\title{
Development of Henan Power Video Quality Monitoring and Analysis System
}

\author{
CUI Zhi-qiang, LI Lu-yuan, LI Yong-jie, XU Dong-jiao, CHANG Ying, WANG \\ Xin
}

Information \& Telecommunication Co. of State Grid Henan Electric Power Company, Zhengzhou 450052, China

Key words: video quality; monitoring and analysis; conference system

\begin{abstract}
Henan power video quality monitoring and analysis system combined with Henan power grid video conference system operation and maintenance reality. The system uses the HUASAN network monitoring module combined with HUAWEI conference network management and conference terminal to adjust the measurement information integrated development. The system can monitor the main influencing factors of video quality changes, record the real-time data of the main equipment running in the conference system, and analyze the situation where the video quality is affected. The system is connected to the test, all the functions are all qualified and runs well, which provides strong support for the stable operation of the Henan power grid video conference system.
\end{abstract}

\section{Introduction}

Henan Electric Power currently deployed several sets of different functions of the conference system. They are the important communications business platform of the company's production and operation. The meetings involve over ten thousand participating units. This is of great significance to Henan power grid.[1-3]At present, the system MCU, terminals, switches and many other equipment, network elements are not included in real-time monitoring When the occasional video quality of the conference TV deterioration, there dropped frames, mosaic and other phenomena, affecting the convening and listening to the meeting. There is no warning that the conference television is affected, and what threshold interference will affect the meeting can not be judged. Due to unknown reasons, the impact of the threshold is unknown, and then can not be targeted to improve and preventive measures. In order to solve the technical problem of video quality fluctuation alarm [4-7], the company's technical staff to consider the development of video quality monitoring and analysis system. The system generates an alarm once it detects a fluctuating video quality. The system remind the operator to troubleshoot the relevant equipment, so that the video quality is no longer deteriorated which will produce mosaic, blurred screen and other issues.

\section{The System Program Introduced}

In this paper, a system is developed, which is based on the HUASAN combination of the network monitoring module and the HUAWEI conference network management and conference terminal. The system can monitor the main influencing factors of the video quality change, record the real-time data of the main equipment running in the conference system, analyze the impact of the video quality, and make a preliminary guide to the fault processing. In this paper, the HUASAN network monitoring module is used to obtain the real-time data of the network, obtain the real-time data and alarm identification of the conference device from the conference device, and comprehensively analyze the data to form the video quality fluctuation alarm.

The platform is divided into four parts: platform construction, data acquisition, data analysis and alarm output. 


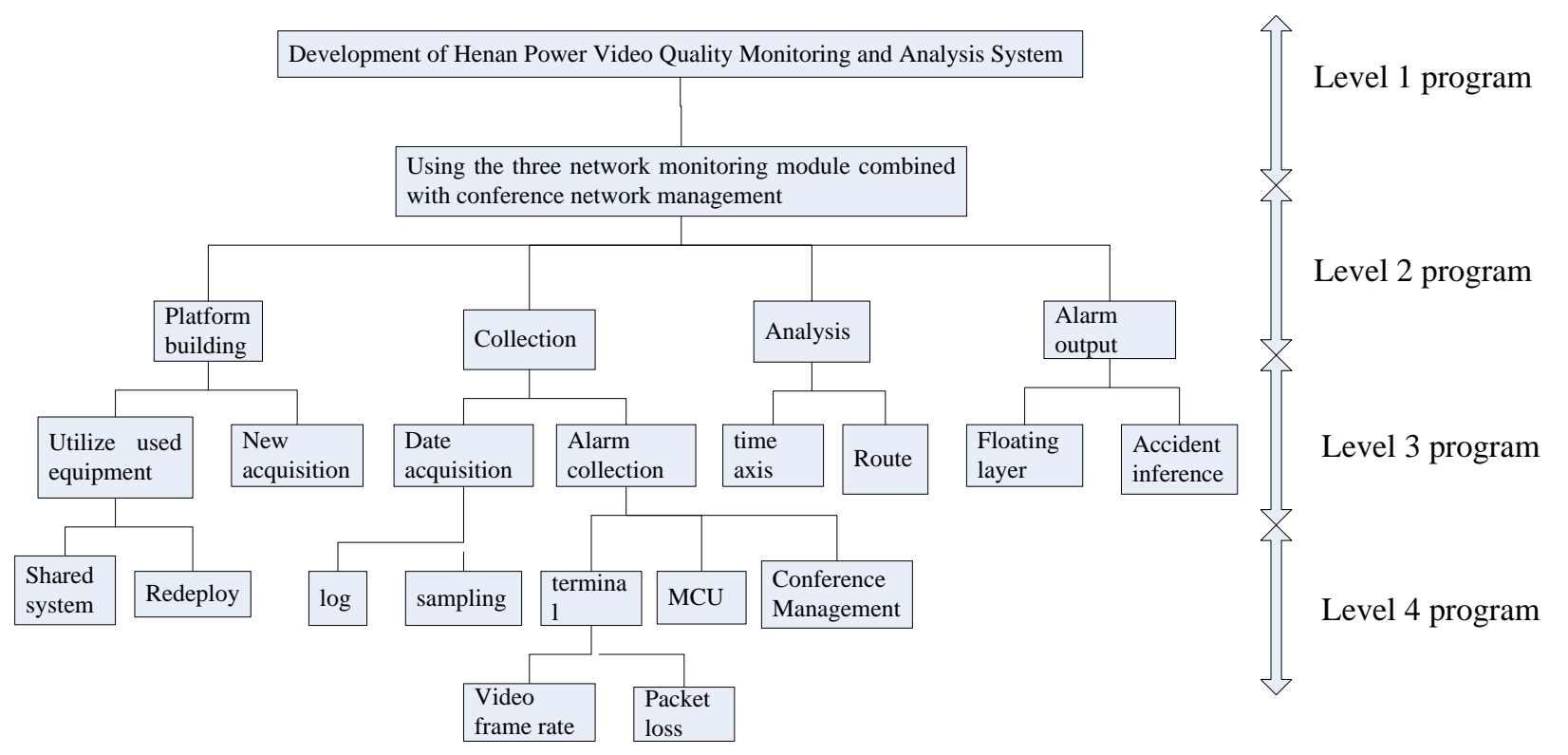

Figure 1 system platform development framework

Platform Building. The use of emergency monitoring standby server 1 and conference network control machine 2 sets up the platform. Server configuration Xeon eight core sixteen thread CUP, $16 \mathrm{G}$ memory, fully meet the needs of the system, greatly saves the investment. It is powerful, conducive to the development and operation. The server has been deployed on the windows server2008, the installation of the MySQL database to meet the development needs of the system. Free space 327G. The interface server function is reserved. The software is complete and the deployment is simple. Retain the original function of the server, easy to restore the original situation in case of emergency. Taking into account the original needs, but also to meet the needs of system development.

Data Collection. The interface tool library automatically receives device logs, obtains device configuration and runs data. Synchronization speed, the average measured delay $<0.4$ seconds. The log has a timestamp, easy to verify accuracy, and facilitate post-data analysis. Data read accurate and verifiable, collection delay is small. Interface tool library to read the terminal web management page, access to the terminal alarm field, the alarm information is simple and accurate. Background read, does not affect the terminal work.

Data Analysis. Routing analysis equipment data and alarm data has been acquired based on the judgment of alarm involved units and equipment, aided by the time axis. Screening and verification of accurate data, there is no association or omission of redundant, auxiliary according to the time axis verification workload is small, fast analysis. A small amount of auxiliary screening.

Alarm Output. Is highlighted on the entire network topology, and the floating layer displays an alarm. The alarm is clear and the location is intuitive. You can view the global alarm on the topology map and check the alarm. Suitable for conference administrators to use, can simultaneously watch a number of alarm brief, in line with hidden habits, easy to use. The video quality analysis software MSU Video Quality Measure is introduced, and the video and local video recordings are compared and analyzed under the experimental conditions. The video quality index, record packet loss rate and video frame rate change are recorded, and the comparison file is formed. 


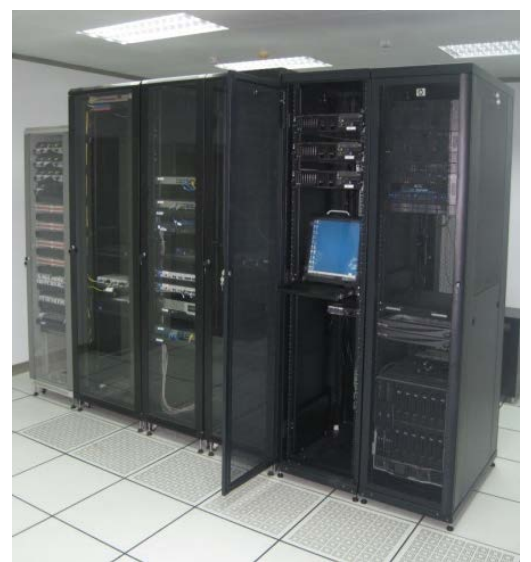

Figure 2 platform implementation

\section{The Implementation of the System Program}

Implementation I:Platform to Build. Established a 5-party meeting in the emergency meeting MCU using the existing network environment of emergency conference system .Put in three 9039S, one TE50, four Test terminal of the conference and one recording and broadcasting server. Four Test terminal simulate the various units participating the conference. Respectively, use the IP segment address of province company, zhengzhou company, Transmission and distribution company. The recording and broadcasting server is used to keep the test video. Simulated bandwidth fluctuations, delay changes, channel quality interference by means of downloading large files, routing interference and so on. Then collect and collate the relevant data. Our teammates inspected the hardware of platform every day, and checked the log and performance indicators mainly from trouble-free time, cpu utilization and other aspects. As of October 7, the platform did not occur outage, to meet the trouble-free time is greater than 30 days requirements. The average CPU carrying capacity is $12.66 \%$ in 60 days, to meet the requirement of load rate less than $40 \%$.

Implementation II: Data Collection. Collection of Network Element Information. Configured log push service for the experimental network equipment, and deployed the log receive client on the server.

Extract the timestamp within the information when receiving the data. The data which sequence corrected is written to the database. Otherwise the device is required to retransmit at the next transmission.

Collection of Alarm Information. Extracted the alarm identification information from the terminal web management page when background log in.

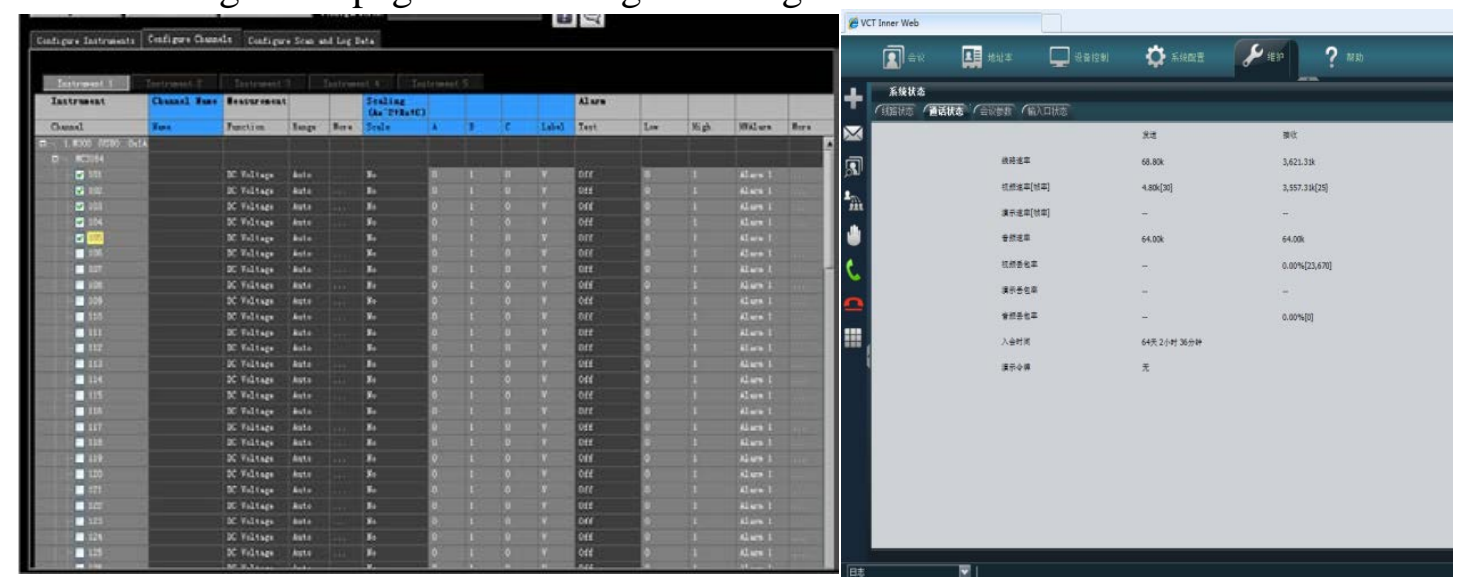

Figure 3 data table screenshot

Implementation III: Data Analysis. Extracting the time stamp of the obtained packet loss rate. To compare the same routing equipment configuration and conditions which has been obtained in the database using the time as a pointer. Filter the device information that parameter change over threshold, and push to the alarm release. In order to ensure the accuracy of the alarm information, 
unified the whole network equipment clock using the time signal as benchmark released by Conference system MCU clock.

The layout of the page content and the various functions was discussed. A publishing page sketch was drawn. The page is divided into device list area, alarm list area and device topology. When the alarm is pushed, you can pop up the alarm box to display the alarm details when click on the alarm. And the device is highlighted with the parameter change when the alarm occurs. The page updates the topology and alarm information in real time according to the alarm data table.
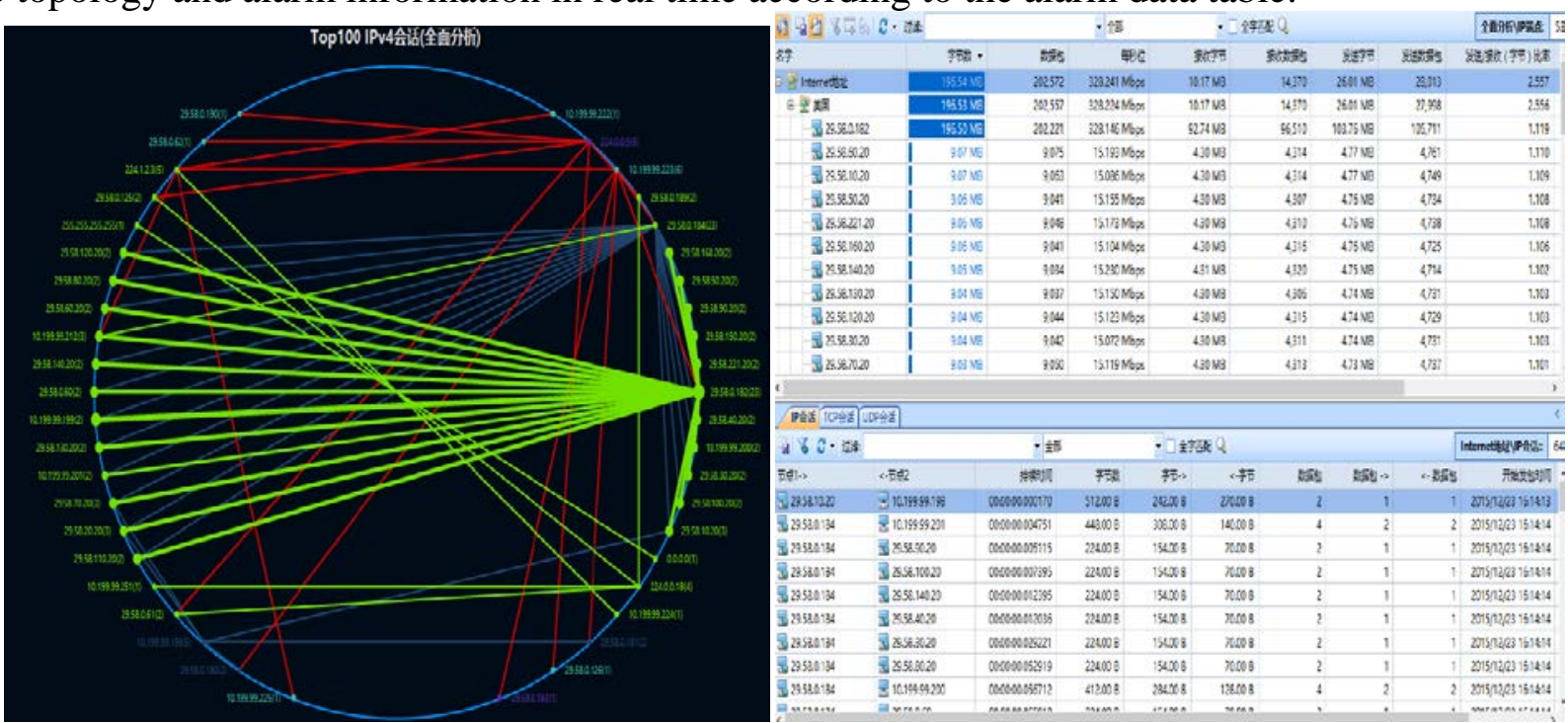

Figure 4 system page screenshots

Implementation IV: Alarm Output. The alarm output interface was designed where the entire network topology device is highlighted, and the floating layer shows the details of the program.

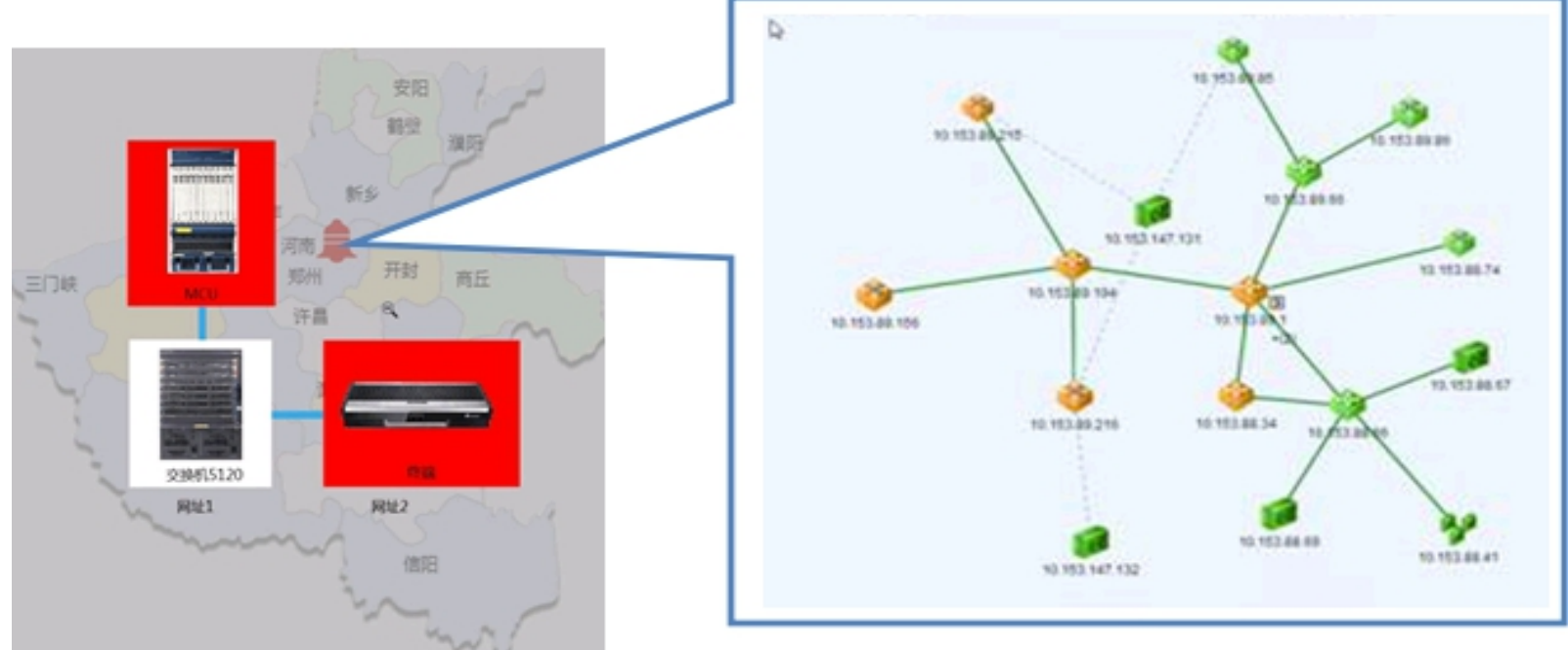

Figure 5 Alarm output interface

Implementation V: System Testing. The video quality monitoring and analysis platform was accessed to the experimental system After the development of the platform. Review the alarm changes when change the experimental network parameters and topology in order to causing video quality fluctuations.

The video quality monitoring and analysis platform was successfully accepted by the Information and Communication Company of State Grid Henan Electric Power Company In mid-October. It was accessed to the provincial company emergency high-definition video conferencing system to conduct field verification. After the system was used, recorded 19 times video quality fluctuation (packet loss rate less than 5\%), 6 times alarm video quality fluctuation event, the actual video quality is disturbed 6 times, and video quality fluctuation event alarm rate is $100 \%$. 
Table 1 System running test data

\begin{tabular}{|c|c|c|c|c|}
\hline \multirow{2}{*}{$\begin{array}{c}\text { Serial } \\
\text { number }\end{array}$} & Check time & $\begin{array}{c}\text { Run error } \\
\text { number }\end{array}$ & $\begin{array}{c}\text { The total number } \\
\text { of runs }\end{array}$ & Error rate \\
\cline { 2 - 5 } & 10.5 & 19 & 46731 & $0.04 \%$ \\
\hline 2 & 10.11 & 55 & 106722 & $0.05 \%$ \\
\hline 3 & 10.18 & 143 & 170512 & $0.08 \%$ \\
\hline 4 & 10.24 & 201 & 291043 & $0.07 \%$ \\
\hline 5 & 11.1 & 316 & 560017 & $0.06 \%$ \\
\hline 6 & 11.7 & 377 & 1026672 & $0.04 \%$ \\
\hline 7 & 11.19 & 419 & 1613276 & $0.03 \%$ \\
\hline
\end{tabular}

\section{Conclusion}

Henan power video quality monitoring and analysis system combined with Henan power grid video conference system operation and maintenance reality. The system uses the HUASAN network monitoring module combined with HUAWEI conference network management and conference terminal to adjust the measurement information integrated development. The system can monitor the main influencing factors of video quality changes, record the real-time data of the main equipment running in the conference system, and analyze the situation where the video quality is affected. The system is connected to the test, all the functions are all qualified and runs well, which provides strong support for the stable operation of the Henan power grid video conference system.

\section{References}

[1] Li Yongjie, Chang Ying, Wang Xin, etc. Henan TV video conference television system architecture and analysis [J]. Television Technology, 2014, 38 (19): 221-224.

[2] Huang Yuhui, Wang Yan, Pan Jie. Television video signal quality evaluation [J]. Quality and standardization, 2007 (8): 28-30.

[3] Gu Chong, Si Zhanjun. Application of video quality evaluation method [C] // 2012 China Printing and Packaging Academic Conference.

[4] Wei Xuehui. Image and video quality of a number of studies [D]. Zhejiang University, 2008.

[5] Yu Jing, Zhou Guoyao, Hu Y. Method and system for video quality detection: CN, CN 103281559 A [P]. 2013.

Design and Development of Video Quality Detection System for Video Surveillance [D]. Huazhong Normal University, 2011.

[7] Liang Zhimin. Video image quality detection technology research and practice [D]. Beijing University of Posts and Telecommunications, 2012. 